\title{
BEST receiver experience: general architecture, design and integration
}

\author{
F. Perini ${ }^{1}$, G. Bianchi ${ }^{1}$, M. Schiaffino ${ }^{1}$, and J. Monari ${ }^{1}$ \\ INAF-Istituto di Radioastronomia, Via Fiorentina 3508/B, Medicina (BO), Italy \\ f.perini@ira.inaf.it g.bianchi@ira.inaf.it mschiaffino@med.ira.inaf.it j.monari@ira.inaf.it
}

Abstract. The receiver for the SKADS/BEST pathfinder is a low cost, low noise, high dynamic range, easily replicable and highly integrated block up to the data acquisition. The aspects related to the design of such a receiver including mechanics (housing and integration), setting control and the local oscillator network are here reported.

\section{Introduction}

The Medicina technical group designed and constructed the blocks of the BEST demonstrators (they are a part of the Northern Cross radiotelescope), to produce technology and to verify the basic SKA concepts. Possible low cost solutions for crucial system blocks such as front ends, analogue optical links, sync and LO distributors, IFs circuitry, wide-band, high dynamic range high speed data acquisition and processing systems have been investigated and realized.

\section{BEST-2 antennas and front-end boxes}

The focal line of the BEST-2 system, implemented in the N/S arm of the Northern Cross Radiotelescope, is composed by 64 dipoles electrically connected in parallel in groups of 16 on 4 different segments of the differential line. These four differential fragments are converted into single lines by means of 4 baluns connected to a impedance adapters. The four different electrical signals are successively carried out, through watertight connectors to four waterproof boxes designed to contain one front-end board, one electro-optical converter, one protection circuit and one optical fibre collecting tray (Figure 1). A canalization structure is built to carry optical fibres and power supply cables on the focal-line (Figure 2).

The BEST-2 front-end (Figure 3) performances are:

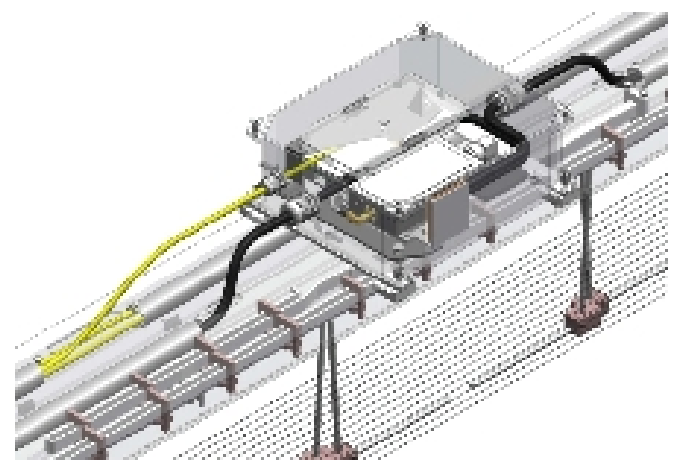

Fig. 1: BEST-2 front-end box on the top of the focal line

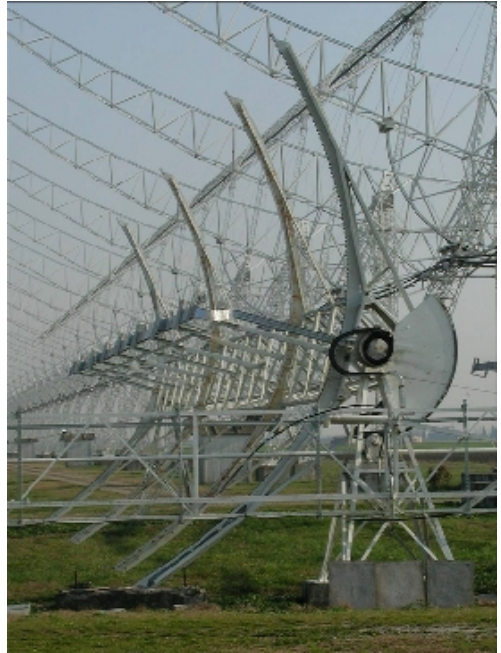

Fig. 2: A North-South cylinder

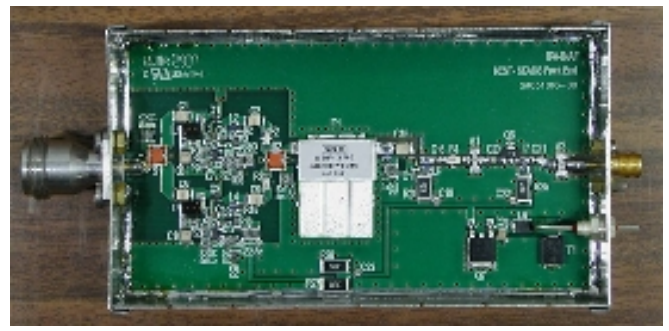

Fig. 3: BEST-2 Front-End

- $\mathrm{NF}=0.45 \mathrm{~dB}, \mathrm{Tn}=32 \mathrm{~K}$

- $\mathrm{Gain}=60 \mathrm{~dB}$

- $\mathrm{BW}=16 \mathrm{MHz} @ 408 \mathrm{MHz}$

- OIP3 > +33 dBm

- InputRL $>15 \mathrm{~dB}$

- OutputRL > $15 \mathrm{~dB}$

- PowerSupply = (10 - 15) Volt @ $245 \mathrm{~mA}$ 


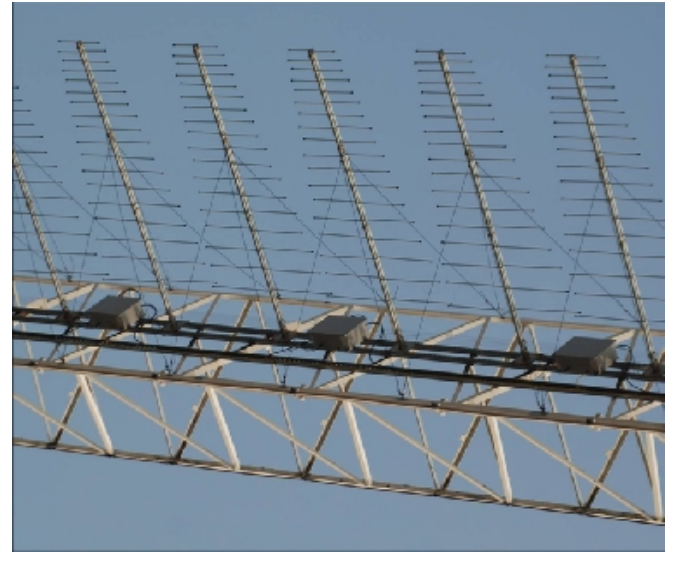

Fig. 4: East-West arm with Log-Periodic antennas installed

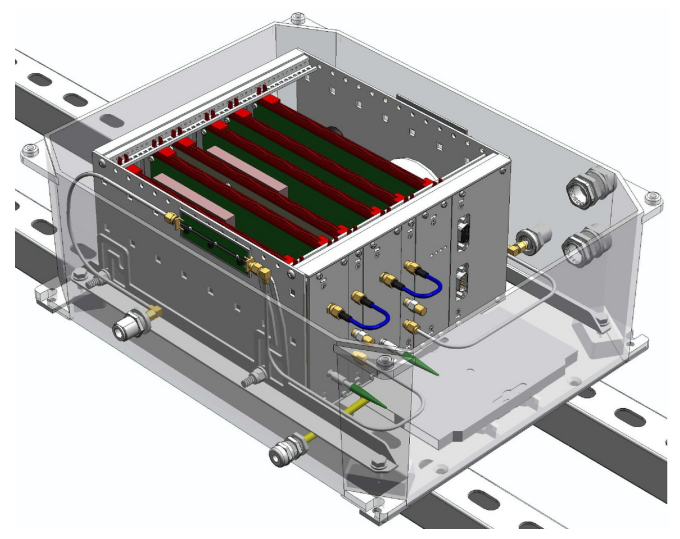

Fig. 5: BEST-31o Front-end box on the top of the focal line

\section{BEST-3lo antennas and front-end boxes}

The focal line of the BEST-3lo system, implemented in the $\mathrm{E} / \mathrm{W}$ arm of the Northern Cross Radiotelescope, is composed by an array of $18 \mathrm{log}$ periodic antennas (see Fig. 4) connected via coaxial cables to waterproof front-end boxes containing two front-end boards, two optical transmitters with antivibration system, one micro-controller and one power supply board (Figure 5).

A canalization structure is realized on the antennas body to carry optical fibres and power supply wiring to the receiving room.

The Best-3lo front-end (Fig. 6) performances are:

- $\mathrm{NF}=1.35 \mathrm{~dB} @ 180 \mathrm{MHz}, \mathrm{Tn}=105 \mathrm{~K} @ 180 \mathrm{MHz}$

- Gain = $50 \mathrm{~dB}$

- $\mathrm{OIP} 3=+25 \mathrm{dBm}$

- Switchable filter bank

- FM-TV band stop filter

\section{Optical link}

In order to obtain the best performances in term of flatness and equalization between the channels, an architecture based

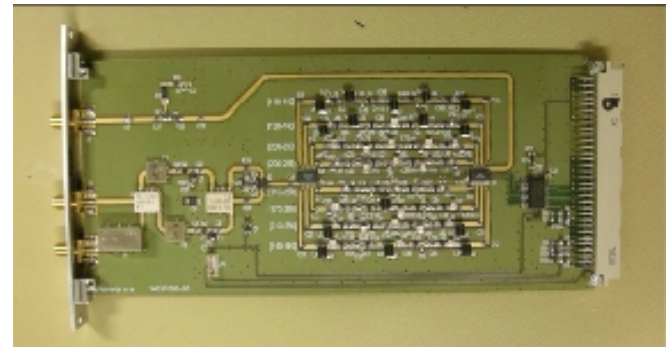

Fig. 6: BEST-3lo Front End

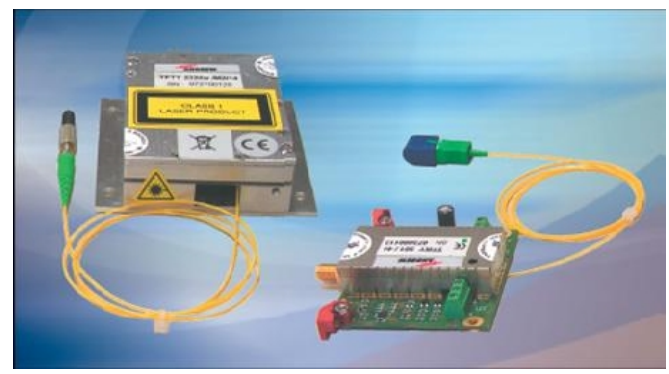

Fig. 7: Customized Andrew’s Analogue optical link

on analogue optical transmission has been implemented.

Further studies of reliability have demonstrated that optical links is preferable respect to coaxial cables one. Several optical analogue links were studied for the BEST system. Finally an Andrew customized version was installed for both systems (Fig. 7). The main specifications are listed below:

- $\lambda=1310 \mathrm{~nm}$

- $\mathrm{NF}=36 \mathrm{~dB}$

- Gain $=-25 \mathrm{~dB}$

- $\mathrm{BW}=(0.1-1) \mathrm{GHz}$

- IIP3 > +33 dBm

- InputRL > $15 \mathrm{~dB}$, OutputRL $>20 \mathrm{~dB}$

- PowerSupply=(12-15) Volt @ 75 mA

\section{Control system for IF receivers}

In order to have an easy-replicable system for each receiving chain without changing the control software and avoiding the replication of the efforts, we designed and developed a general purpose board called "carrier". It is based on microcontroller ATMEGA169-16AI and it allows to control and acquire ancillary data from the various $\mathrm{RF} / \mathrm{IF}$ sections. The carrier board permits to be connected via LAN or RS485 by a dedicated backplane and it generates all the I/O necessary to control the RF/IF circuitry (i.e. switches), acquire analogue signals (i.e. Total Power) and it allows to power-on/off each active device by photo-MOS. The board was designed and prepared to be carried on a metallic milled box ensuring the necessary shielding to the RF analogue signals during the switching of the digital components (Fig. 8). 


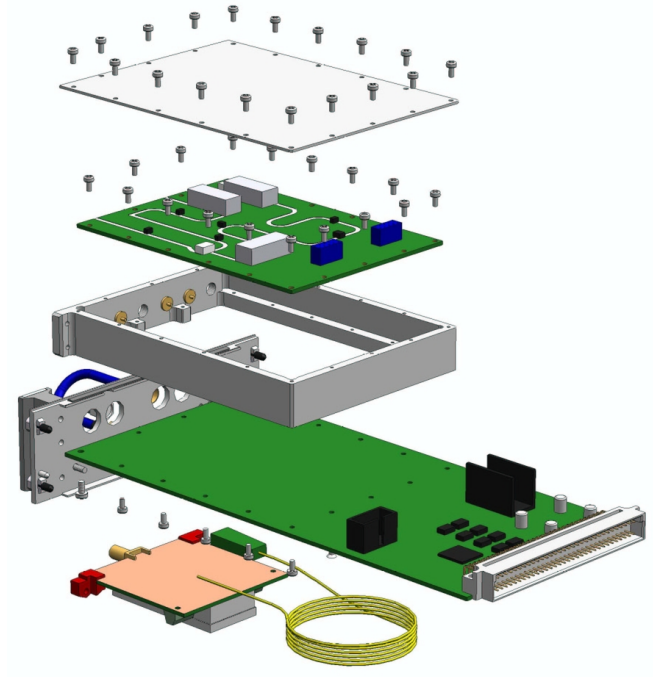

Fig. 8: Assembly of IF + Carrier Board + Optical RX board

\section{IF systems for BEST-2 and BEST-3lo}

The designed receiver architectures for BEST-2 and BEST-3lo are respectively a single conversion to $\mathrm{IF}=30 \mathrm{MHz}$ and a direct amplification without any conversion. All the RF chains are directly installed on the carrier board that control digital attenuator, RF switches and all the supplies of actives devices. The receiver gain is calculated in order to obtain the best power at the input of the Berkeley ADC, thus Pin(ADC) $=-40 /-$ $30 \mathrm{dBm}$. Because BEST-3lo system has a variable bandwidth depending on the filter selected at the front end level, a digital attenuator is provided in order to tune the power at the output of the board.

The main specifications of the BEST-2 RF/IF (first group, see Fig. 9), and BEST-3lo receiver (second group, see Fig. 10), are listed below:

- IIP3 > +16 dBm

- $\mathrm{IF}=30 \mathrm{MHz}, \mathrm{OL}=378 \mathrm{MHz}$

- $\operatorname{Gain}_{\mathrm{RX}}=(16-47) \mathrm{dB}$

- Gain $_{\mathrm{RX}}$ step $=0.5 \mathrm{~dB}$

- $\mathrm{IIP} 3>-10 \mathrm{dBm}$

- $\mathrm{RF} / \mathrm{IF}=(120-240) \mathrm{MHz}$

- $\mathrm{Gain}_{\mathrm{RX}}=(13-45) \mathrm{dB}$

- Gain $_{\mathrm{RX}}$ step $=0.5 \mathrm{~dB}$

\section{Local oscillator, clock and sync distribution}

A splitter chain architecture has been used to distribute LO, Clock and Sync signals to the IF circuitry and data acquisition

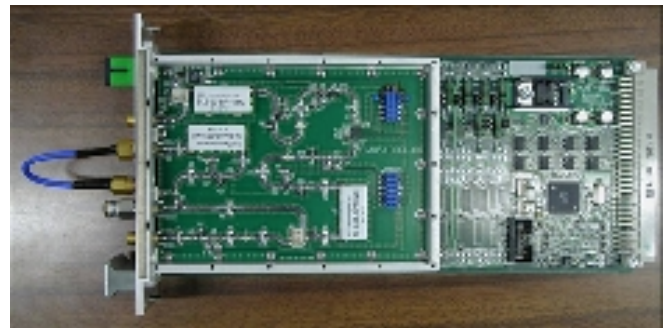

Fig. 9: BEST-2 IF board

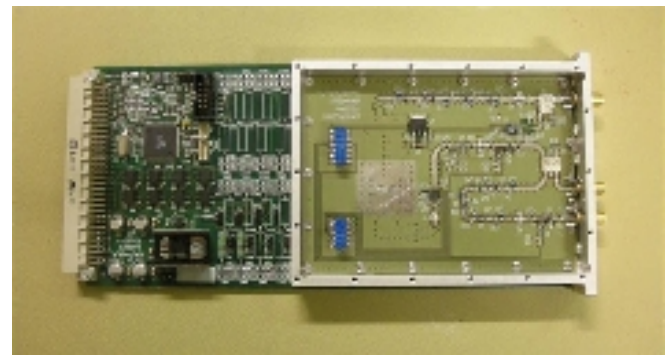

Fig. 10: BEST 3-lo IF board

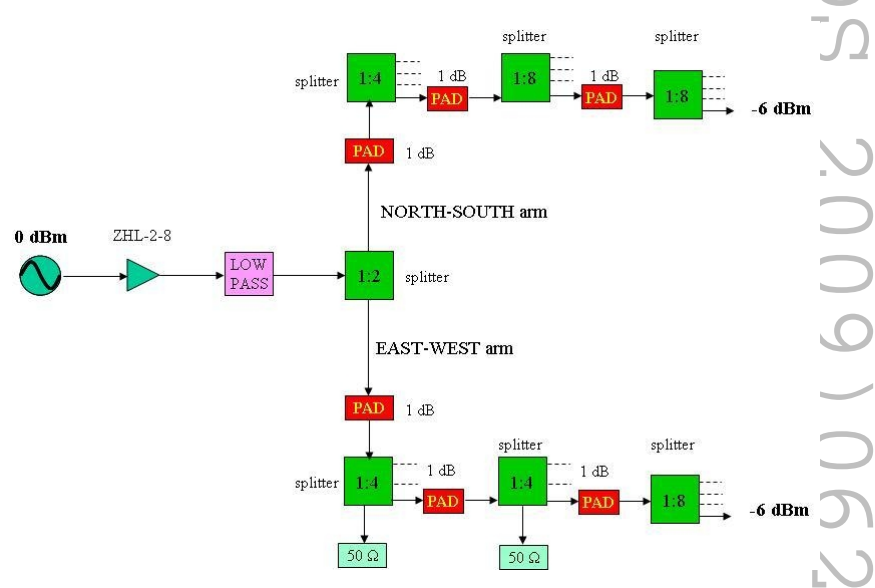

Fig. 11: Schematic block diagram of the clock distributor

system. The block diagrams are shown in the Fig. 11 (Clock) and Fig. 12 (LO). The LO distributor and racks cabling are shown in Fig. 13. To synchronize the data acquisition boards, based on a Berkeley University A/D-iBOB and Bee2 systems, a low jitter PPS (Pulse Per Second) distributor unit (Fig. 14) was designed and tested on the BEST system.

\section{The shared digital back-end}

The BEST acquiring and back-end system is based on iBOB/BEE2 Casper modular architecture. This can be alternatively connected to BEST-2 or BEST-3lo receiving chain by a switching analogue matrix (Fig. 15). 


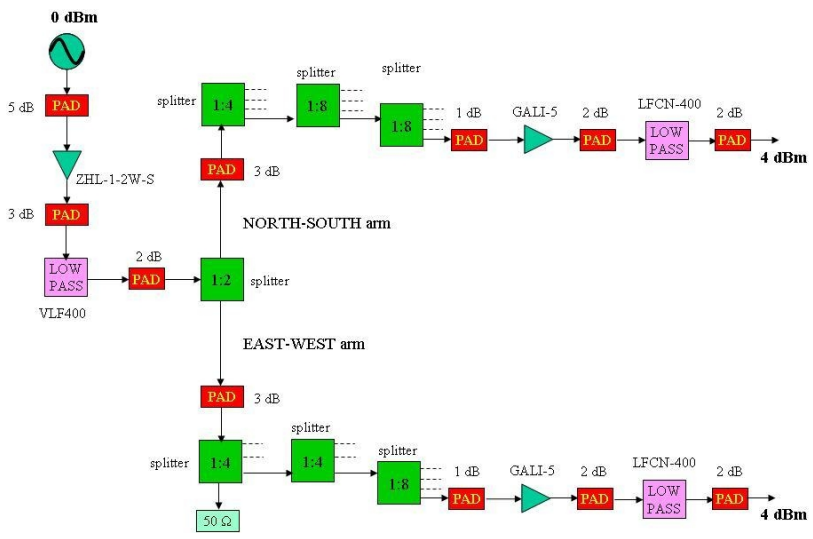

Fig. 12: Schematic block diagram of the LO distributor

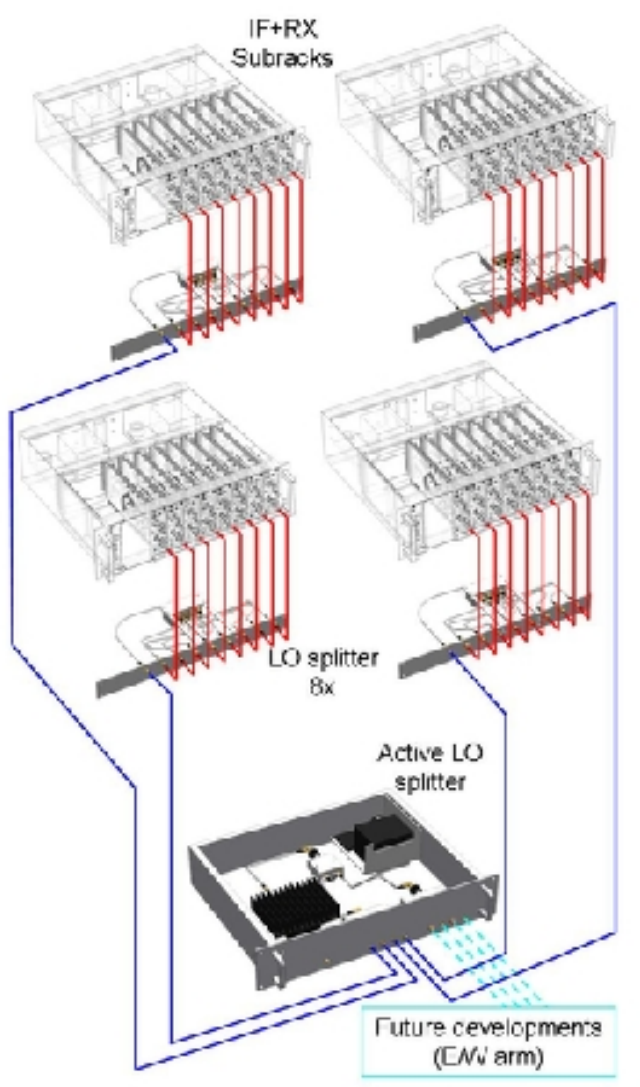

Fig. 13: LO distributor: amplifier, E/W - N/S splitting blocks and IF + RX subracks

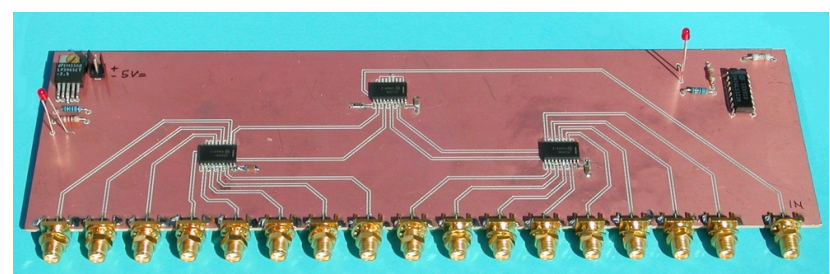

Fig. 14: PPS distributor board

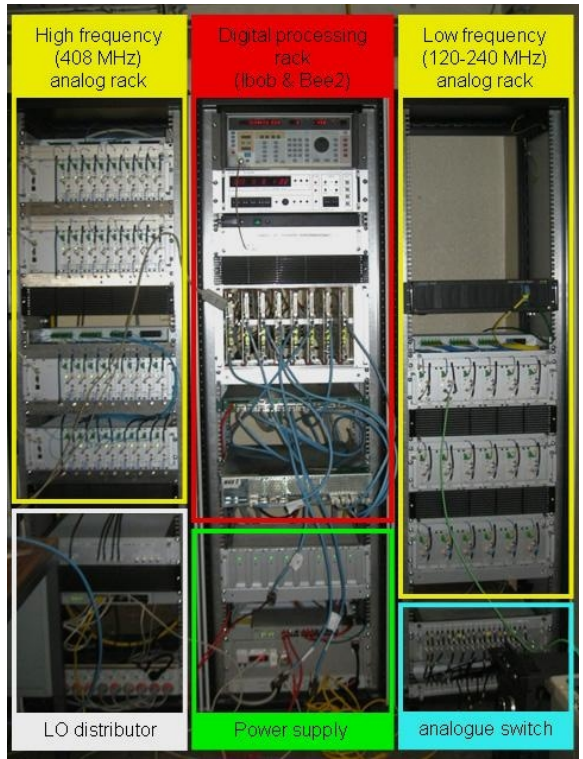

Fig. 15: BEST back end. On the left the BEST-2 circuitry and on the rigth the BEST-31o electronics

\section{Conclusions}

The experience gained by the Medicina technical group in BEST-2 $(\mathrm{Fc}=408 \mathrm{MHz}, \mathrm{BW}=16 \mathrm{MHz})$, BEST-3lo $(\mathrm{Fc}=$ $180 \mathrm{MHz}, \mathrm{BW}=120 \mathrm{MHz}$ ) system, led to design a replicable and reliable shared architecture between the antennas and the receiving room. Both solutions are based on an high dynamic, low noise, low cost front end connected by an analogue fibre optic link to the receiving room. Here was installed a compact IF system controlled by a general purpose common board called Carrier board. The iBOB/BEE2 Casper acquiring and back-end system, can be alternatively connected to BEST-2 or BEST-3lo receiving chain.

For the SKA framework this could be the line to follow in order to avoid the dispersion of efforts and to obtain high performance, high reliability and low cost general receiving architecture. 